\title{
Prediction of Cutting Temperature in the Milling of Wood-plastic Composite Using Artificial Neural Network
}

\begin{abstract}
Feng Zhang, ${ }^{a}$ Zhanwen $\mathrm{Wu},{ }^{\mathrm{b}}$ Yong Hu, ${ }^{\mathrm{b}}$ Zhaolong Zhu, ${ }^{\mathrm{c}, \mathrm{d}, *}$ and Xiaolei Guo ${ }^{\mathrm{a}, \mathrm{c}, *}$
In the milling of wood-plastic composites, the cutting temperature has a great influence on tool life and cutting quality. The effects of cutting parameters on the cutting temperatures in the cutting zone were analyzed using infrared temperature measurement technology. The results indicated that the cutting temperature increased with the increase of spindle speed and cutting depth but decreased with the increase of feed rates. In addition, based on experimental data, a BP neural network model was proposed for predicting the cutting temperatures. The value of $R^{2}$ was 0.97354 for the testing data, which indicates that the developed model achieved high prediction accuracy, respectively. The results of the study can play a guiding role in the prediction and control of cutting temperature, which is of great importance in the improvement of tool life, machining quality, and machining efficiency.
\end{abstract}

Keywords: WPC; Cutting temperature; BP neural network

Contact information: a: College of Materials Science and Technology, Nanjing Forestry University, Nanjing 210037, China; b: Bosun Tools Co., Ltd., Shanghai 201300, China; c: Co-Innovation Center of Efficient Processing and Utilization of Forest Resources, Nanjing Forestry University, Nanjing 210037, Jiangsu, China; d: College of Furnishings and Industrial Design, Nanjing Forestry University, Nanjing 210037, China; *Corresponding authors: njfuzzlong@outlook.com; guo.xiao.lei@hotmail.com

\section{INTRODUCTION}

Wood fiber/polyethylene composite (WPC) is a relatively new type of material mainly composed of poplar fiber (thermal conductivity is $0.2 \mathrm{~W} /(\mathrm{m} \cdot \mathrm{k})$ ) and polyethylene (thermal conductivity is $0.42 \mathrm{~W} /(\mathrm{m} \cdot \mathrm{k})$ ) (Malkapuram et al. 2009; Ramesh et al. 2018). Because of the combination of the double advantages of wood fiber and plastic materials, WPCs exhibit excellent comprehensive properties (Gardner and Bozo 2018; Ma et al. 2020), such as high strength, high impact resistance, dimensional stability, and good wear resistance (Kumar et al. 2011). Moreover, WPC also has the dual processing properties of wood and plastic. They can be formed by extrusion and hot pressing, or processed by wood processing methods such as sawing, planing, rotary cutting, grinding, and sanding. Woodplastic composite materials are widely used in the fields of paneling materials, furniture materials, interior decoration materials, and automobile interiors (Liukko et al. 2007). Most of the wood-plastic products need to be machined (milling, drilling, grinding, or sawing) to meet the requirements of shape tolerance and surface roughness, and milling processing is an important way to obtain WPC end products (Somsakova et al. 2012; Guo et al. 2021).

Most of the energy consumed during WPC milling is converted into heat, which is gathered in the cutting area (Karaguzel et al. 2016). However, different from metal cutting, coolant cannot be used in WPC milling. The cutting heat generated during WPC milling can only be dissipated by the tool, workpiece, and chip (Soler et al. 2015). 
The accumulation of cutting heat leads to an increase in temperature in the cutting region, and excessive temperatures can cause softening of plastics in WPC, which causes the plastic to stick to the tool. Therefore, the increase in cutting temperature will reduce tool life and lead to an increase in the number of tool changes and eventually increase the economic cost (Guo et al. 2021). Furthermore, the heat in the cutting area will affect the quality of the WPC machined surface. The results indicate that the cutting temperature increases and the tool wear area increases as the cutting length increases. In the milling of high-density fiberboard (HDF), Zhu et al. (2017) point out that high cutting speed, which includes high spindle speed and high feed rate, can increase the processing capacity, but it will bring more cutting heat and force to the tool surface at the same time. Guo et al. (2018) stated that more severe cyclical thermal loads and mechanical loads during high-speed milling will cause cracks and fractures in the coating, which will further reduce the life of the tool. Reducing the cutting temperature is the key to achieving high-quality processing of wood-plastic composites for different processing conditions and processing requirements. Hence, it is essential to research the cutting temperature in the milling process of $\mathrm{W}$.

The results of the study by Pei et al. (2016) showed that the cutting temperature increases with the increase of spindle speed and cutting depth, but decreases with the increase of feed rates. However, cutting parameters were selected higher than the usual ones. Based on the experimental data, regression analysis, which is a type of black-box method, can effectively solve the non-linearities in the cutting process. Sreejith et al. (2007) studied the influence of machining conditions on cutting temperature by establishing a multiple regression model. The response surface method (Liyana-Pathirana and Shahidi 2005; Ba and Boyac 2007; Gunst 2008) fits the functional relationship between the factor and the response value using multiple quadratic regression equations. Zhu et al. (2020) used the BBD response surface method to develop mathematical models for cutting forces and temperatures to find correlations between actual and predicted results, which can be used to make accurate predictions. The cutting process of wood-plastic composites can be seen as a complex non-linear system (Nie and Bangyan 2009; Gong et al. 2012; Cao and Guo 2013).

It is difficult to accurately establish models with mathematical methods because the state equations of these systems are complicated. The BP neural networks can approach arbitrary non-linear continuous functions by selecting the appropriate weights and structure (Patan 2019; Chen et al. 2020; Wang et al. 2020). Under this situation, a BP neural network can be built to express the nonlinear system of WPC cutting. There have been various publications dealing with applications in the prediction of metal cutting temperature (Fuat et al. 2016; Mia and Dhar 2016), but no application in the study of cutting temperature of WPC.

As observed from the literature review, despite the important influence of cutting temperature on the tool life and the machining quality of WPC, there has been a lack of investigations on the cutting temperature of WPC. The objective of this work was to investigate the effects of cutting parameters on the cutting temperature and establish a prediction model for cutting temperature. Cutting temperatures were measured through the single factor cutting test of these WPCs. The effect of cutting parameters on the cutting temperature was determined by analyzing experimental values. Based on the experimental values, BP neural network was built to predict the cutting temperature of WPC during the milling process. 


\section{EXPERIMENTAL}

\section{Experimental Condition}

A diagram of the experimental set-up is shown in Fig. 1. The cutting temperature test in the milling of WPC was conducted in a computerized numerical control (CNC) machining center (MGK01, Nanxing Machinery Co., Ltd., Guangzhou, China). Infrared imaging (Thermo Vision A20, Thermo Fisher Co. Ltd., USA) was used to measure the cutting temperature during the cutting process. In this device the distance between the camera and the workpiece is $30 \mathrm{~cm}$, and the observable range is $2 \mathrm{~cm} \times 2 \mathrm{~cm}$. The experimental conditions most used in the actual production process are listed in Table 1 and were used for analyzing the influence of cutting parameters on cutting temperature and establishing predictive models.

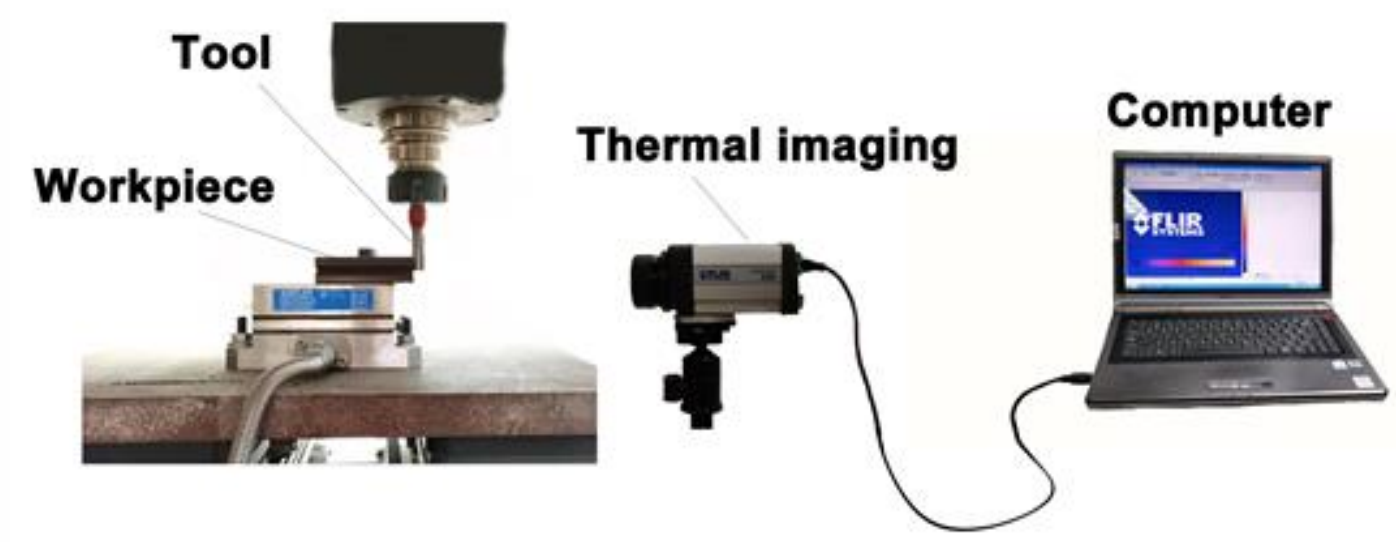

Fig. 1. Experimental set-up

Table 1. Experimental Conditions

\begin{tabular}{|c|c|}
\hline Workpiece Material & Wood-plastic Composite \\
\hline Milling tools & Cemented carbide \\
\hline Spindle speed $(n, \mathrm{n} / \mathrm{min})$ & $6000,8000,10000,12000$ \\
\hline Feed rate $(U, \mathrm{~m} / \mathrm{min})$ & $3,4,5,6$ \\
\hline Cutting depth $(h, \mathrm{~mm})$ & $0.4,0.8,1.2,1.6$ \\
\hline
\end{tabular}

(a)

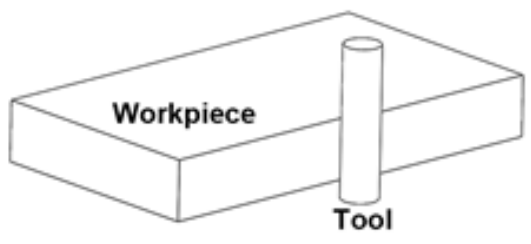

(b)

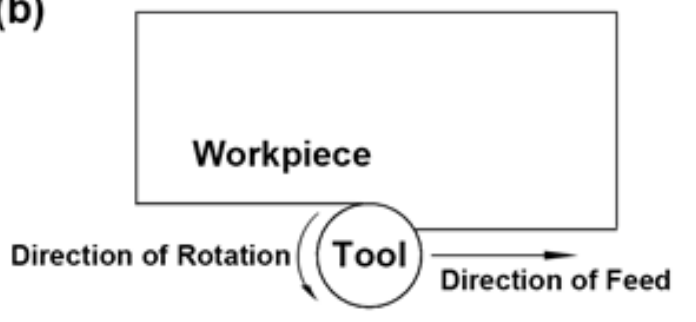

Fig. 2. Schematic diagram of milling 
The workpiece material was a wood-plastic composite (WPC) whose ratio of wood powder to plastic was 7:3. The density of the workpiece was $16.5 \mathrm{~kg} . \mathrm{cm}^{-3}$, and the size of the workpiece was $14 \times 8 \times 2 \mathrm{~cm}^{3}$. Single and straight edge handle milling cutters were used as cutting tools, whose material was cemented carbide. The diameter of tools was 12 $\mathrm{mm}$, and the wedge angle of tools was fixed at $45^{\circ}$. The schematic diagram of the milling process is shown in Fig. 2a, and the relative motion of the workpiece and cutting tool during milling is illustrated in Fig. 2b. The tool rotates at high speed and moves along laterally at the same time. The workpiece is fixed on the workbench.

\section{Temperature Measuring Method}

Infrared imaging, which is a fast and accurate method, was used in this work for measuring the cutting temperature. The cutting temperature area (including workpiece and tool) was measured by infrared thermal imaging (Thermo Vision A20-M, FLIR Systems Inc., Washington D.C., USA) with a refresh rate of $50 \mathrm{~Hz}$. The cutting temperature measuring method is illustrated in Fig. 1. The software ThermaCAM Researcher Professional 2.10 (FLIR Systems Inc., Washington D. C., USA) was used to analyze the information in the captured pictures.

\section{RESULTS AND DISCUSSIONS}

\section{Cutting Temperature Analysis}

In the cutting process, deformation of the material at the tip of the tool (Fig. 4a) and the friction between the rake face and chip is the main source of cutting heat (Fig. 4b), and the secondary source of cutting heat is the friction between the flank face and the workpiece (Fig. 4c). These two types of friction are affected by different cutting conditions, which in turn affect the temperature in the cutting zone. The effects of different cutting parameters (spindle speed, feed rate, and depth of cut) on the average cutting temperature were analyzed to discover the connections between cutting parameters and cutting temperature.

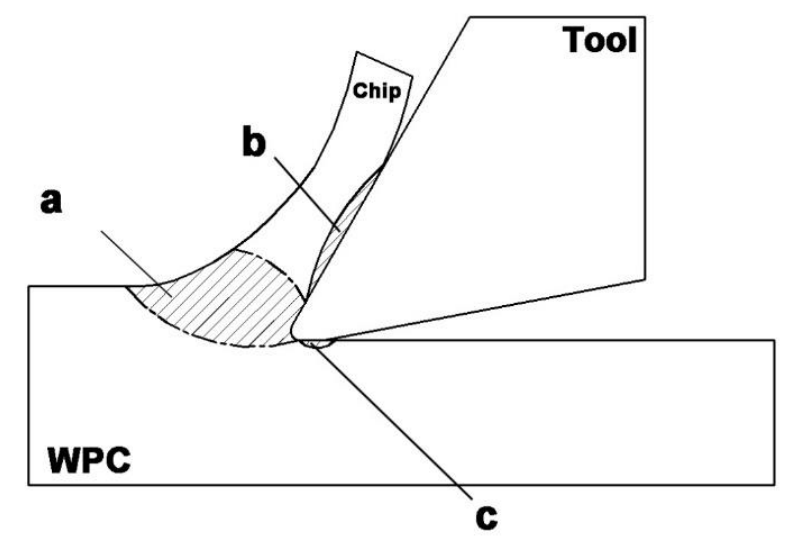

Fig. 3. Schematic diagram of cutting heat area

\section{Experimental Results of Cutting Temperature}

The instantaneous changes in the cutting temperature during the three complete cutting cycles are shown in Fig. 3. It can be found from Fig. 3 that temperature of cutting area rose rapidly at the beginning of cutting, and after some fluctuations, the temperature 
of the cutting area decreased at a slower rate. The change in cutting temperature can be divided into three stages during a cutting cycle. Temperature of cutting area rises rapidly in stage one $(\mathrm{S}-\mathrm{I})$, and the main reason for this phenomenon is the temperature in the first and second deformation zones increases rapidly when the chip thickness increases from 0 to the maximum. In stage two (S-II), the value of cutting temperature fluctuates but is relatively stable. The heat dissipation of wood-plastic composites is poor, the heat generated by cutting is not yet dissipated, and the tool cuts into the workpiece to generate heat. Therefore, the change of cutting temperature is a fluctuating state. In stage three (SIII), when a full cut has been completed, the tool leaves the workpiece, and the cutting temperature decreased at a slower rate. This is mainly because the heat dissipation of woodplastic composites is poor. It is inconvenient to use instantaneous values to analyze the effect of process parameters on temperature because the instantaneous cutting temperature varies considerably during a cutting cycle. Therefore, the average value of the cutting temperature in the stable cutting was chosen to realize the analysis of the effect of cutting temperature.

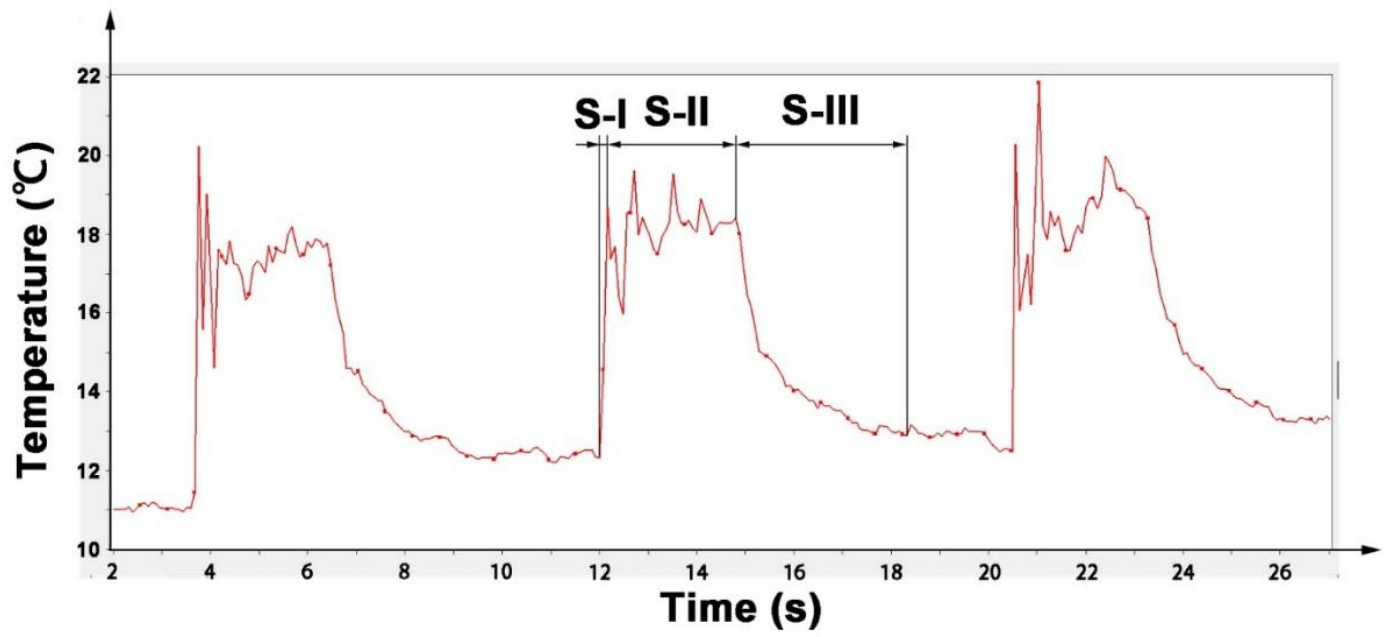

Fig. 4. Experimentally tested cutting temperature changes

\section{Effect of Spindle Speed on Cutting Temperature}

In this study, four different spindle speeds $n$ were adopted to study the influence of spindle speed $n$ on cutting temperature. It can be summarized from Fig. 4 that the cutting temperature increased with the increase of spindle speed. As the spindle speed was increased, the frequency of friction between the rake face and the chip, and the frequency of friction between the flank face and the workpiece increased. Some of the heat will be carried away by the chips and tools, which reduces the cutting heat in the cutting area. However, the increased frictional heat is much greater than the reduced heat of the shear heat source. Because of this, the cutting temperature increases with the increase of spindle speed. 

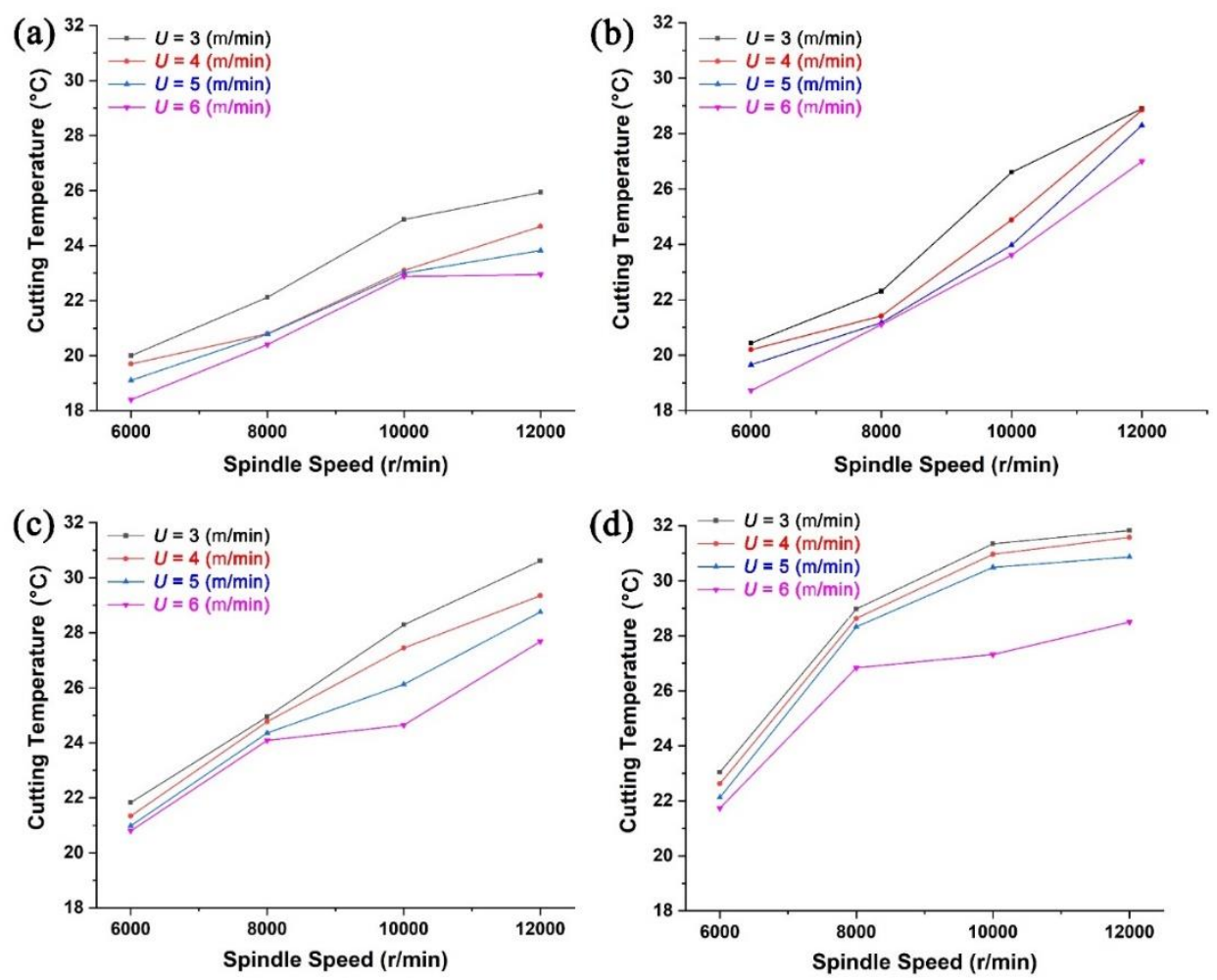

Fig. 5. Effect of spindle speed on cutting temperature at different cutting depth $h$ : (a) $h=0.4 \mathrm{~mm}$; (b) $h=0.8 \mathrm{~mm}$; (c) $h=1.2 \mathrm{~mm}$; (d) $h=1.6 \mathrm{~mm}$
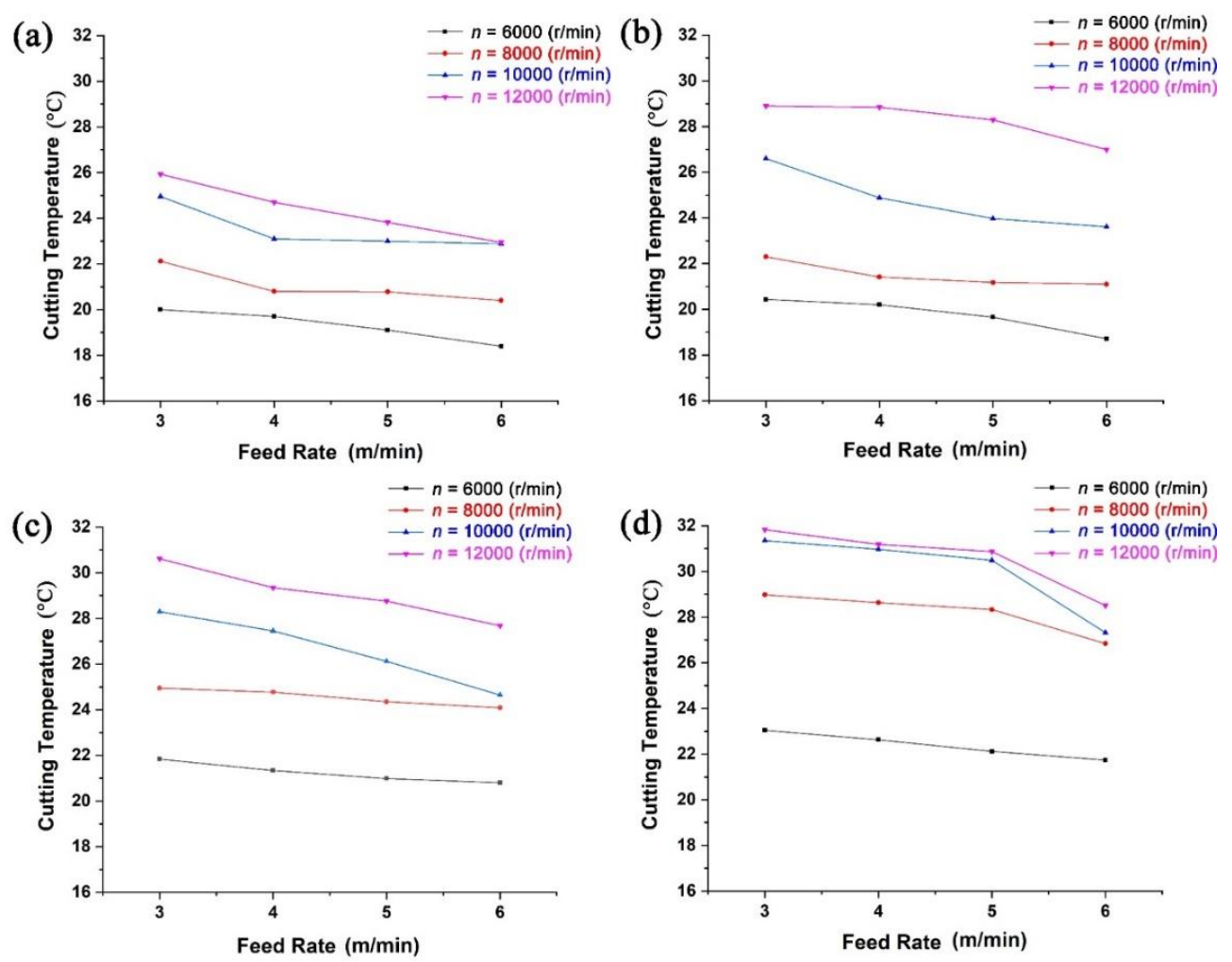

Fig. 6. Effect of feed rate on cutting temperature at different cutting depth $h$ : (a) $h=0.4 \mathrm{~mm}$; (b) $h$ $=0.8 \mathrm{~mm}$; (c) $h=1.2 \mathrm{~mm}$; (d) $h=1.6 \mathrm{~mm}$ 


\section{Effect of Feed Rate on Cutting Temperature}

In this study, four different feed rates $U$ were adopted to study the influence of feed rate $U$ on cutting temperature. It can be concluded from Fig. 5 that the cutting temperature decreased with the increase of feed rates. The thermal conductivity of the tool is higher than that of the workpiece; therefore, the heat transfer into the tool is faster than the transfer to the workpiece. In this case, those other parameters are fixed, and as the feed rate increases, the time that finishes a complete cutting process decreases. As a result, the cutting heat that is transferred into the workpiece is reduced. Because of these factors, the cutting temperature decreases with the increase of feed rates.

\section{Effect of Cutting Depth on Cutting Temperature}

In this study, four different cutting depths $h$ were adopted to study the influence of cutting depth $h$ on cutting temperature. It can be summarized from Fig. 6 that the cutting temperature increases with the increase of cutting depth. In the case that other parameters are fixed, as the cutting depth $h$ increases, the thickness of the chip increases, which in turn leads to a higher pressure between the rake face and the chip. Consequently, the friction between the rake face and the chip becomes more intense. Therefore, the cutting temperature increases with the increase of cutting depth.

(a)

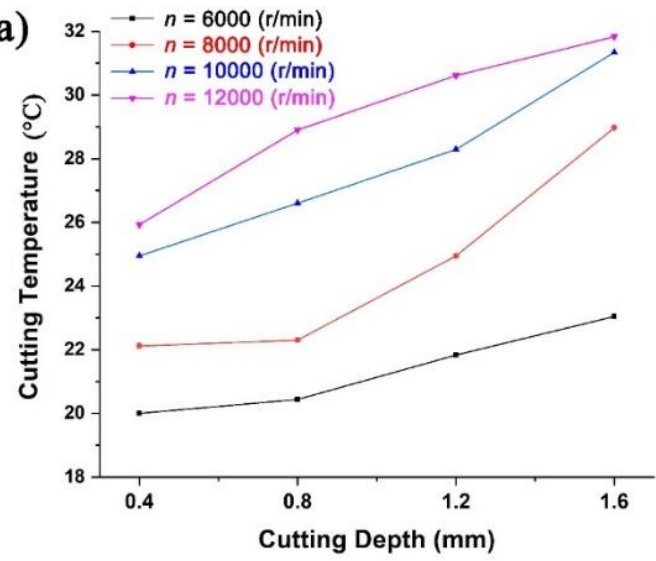

(c)

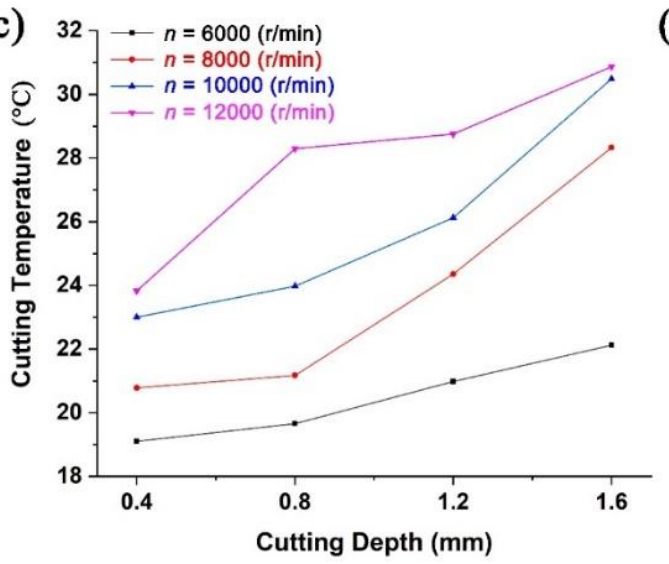

(b)

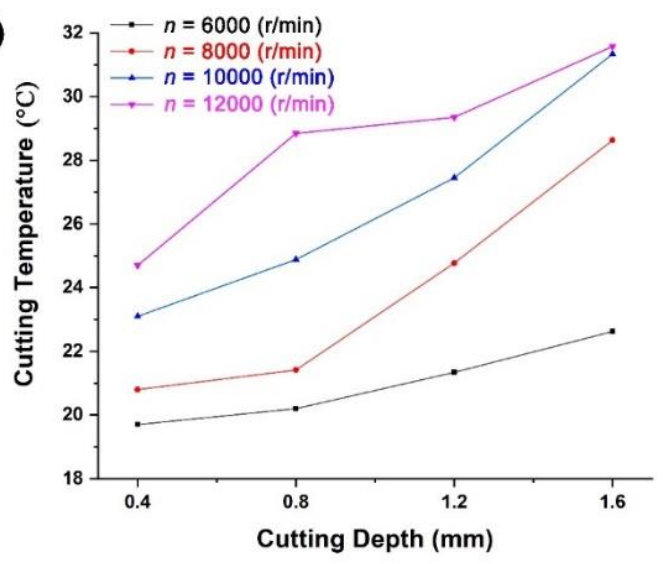

(d)

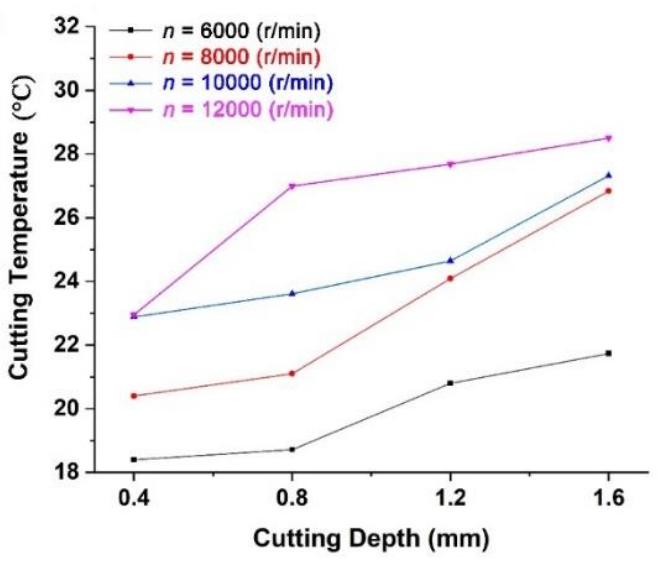

Fig. 7. Effect of cutting depth on cutting temperature at different feed rates $U$ : (a) $U=4 \mathrm{~m} / \mathrm{min}$; (b) $U=5 \mathrm{~m} / \mathrm{min}$; (c) $U=6 \mathrm{~m} / \mathrm{min}$; (d) $U=7 \mathrm{~m} / \mathrm{min}$ 


\section{NEURAL NET ANALYSIS AND FITTING RESULTS}

\section{The Structure of the BP Neural Network}

The structure of the BP neural network used in this paper is shown in Fig. 8. It can be learned that the BP neural network consists of three main layers: input layer, one hidden layer, and output layer. The input layer contains three neural units (spindle speed $n$, feed rate $U$, and cutting depth $h$ ), and the output layer has only one neural unit (cutting temperature). The number of hidden layer units is determined according to Eq. 1,

$$
m=\sqrt{n+l}+\alpha
$$

where $m$ is the number of hidden layer units, $n$ is the number of input layer units, 1 is the number of output layer units, and $\alpha$ is a constant between 1 and 10. In this paper, the value of $\alpha$ is taken as 5, and as a result, the hidden layer contains 7 neural units.

The activation function provides a curvilinear match between input and output layers, and a Log-Sigmoid transfer function has been commonly used as the activation function in BP neural networks ( $\mathrm{Li}$ and Zhang 2017), because it is a differentiable, continuous, and nonlinear function. This function generates a value between 0 and 1 for each value that is entered from the input layer. The equation of the Log-Sigmoid transfer function adopted for the BP neural network in this study is given as Eq. 2:

$$
f(x)=\frac{1}{1+e^{-x}}
$$

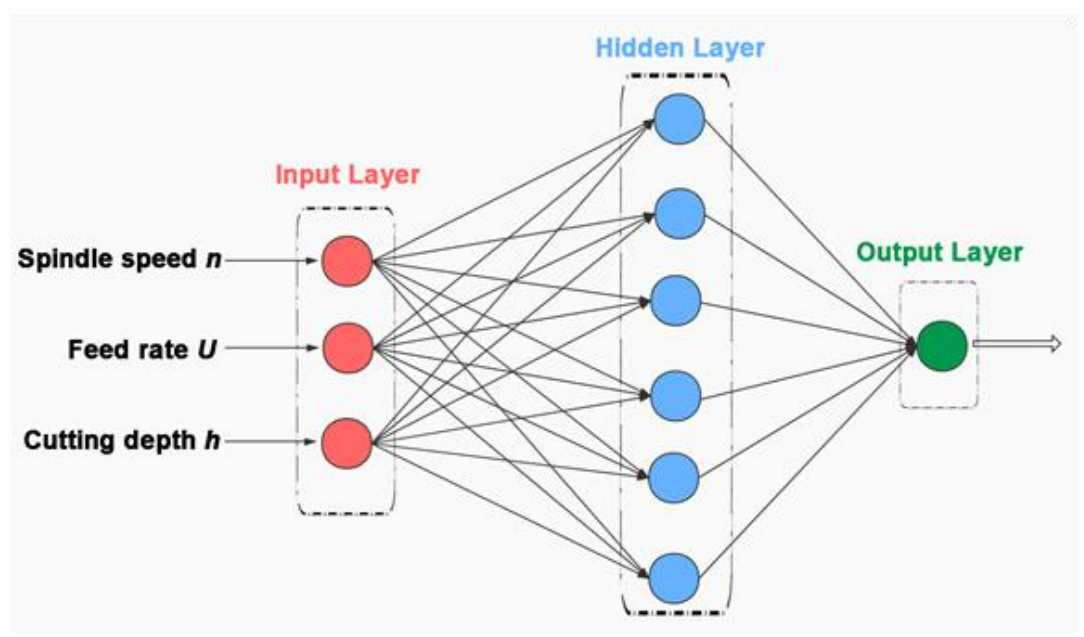

Fig. 8. The structure of the BP neural network

\section{Learning Algorithm}

After determining the number of layers of the BP neural network and the number of neurons in each layer, it is also necessary to determine the weights between the layers. In this research, the random values were first used as weights during training, and then the learning samples were input to obtain the output of the network. After this, the error between the output value and the target value was calculated, and according to which the error was reduced by modifying the weights layer by layer. After several adjustments, the network training was completed when the error reaches the requirement. 


\section{Training and Testing Data}

The selection of training and testing data has an important impact on the accuracy of BP neural network prediction models. In this research, 64 numerical data obtained by experiment were used for the training and testing data for the BP neural network. From these data, 54 groups were randomly selected as training data, and the rest used as test data. In this way, the BP neural network can avoid falling into a local optimal situation.

In this research, the data of BP neural network has different physical importance and magnitude. To avoid large network prediction errors due to large differences in the magnitude of the data, the input and output value were normalized between 0 and 1 , and the function for data normalization were as follows,

$$
x_{k}=\left(x_{k}-x_{\min }\right) /\left(x_{\max }-x_{\min }\right)
$$

where $x_{\mathrm{k}}$ is the pretreatment data, $x_{\max }$ and $x_{\min }$ are the maximum and minimum values of the pretreatment data, and $x_{\max } \neq x_{\min }$ in the pretreatment data.

\section{Statistical Evaluation of Outputs}

To evaluate the developed prediction model, the statistical methods of $\mathrm{R}^{2}$ (absolute fraction of variance) have been used for the comparison. The range of $\mathrm{R}^{2}$ is $[0,1]$, and the closer the value of $\mathrm{R}^{2}$ is to 1 , the more accurate the prediction will be. The coefficient of determination is given as,

$$
R^{2}=1-\sum_{M=1}^{N} \frac{\left(T_{m, M}-T_{p, M}\right)^{2}}{T_{m, M}^{2}}
$$

where $T_{m, M}$ is measured value, $T_{p, M}$ is prediction value, and $N$ is the number of samples.

\section{Prediction of Cutting Temperature Using BP Neural Network}

A computer program for predicting the cutting temperature was developed on the MATLAB (Mathworks Inc., Natick, MA, USA). The spindle speed, the feed rate, and the cutting depth were used in the input layer, and the cutting temperature was used in the output layer. In the program of BP neural networks, the target error of the network net.trainparam. goal was set to 0.001. The learning speed net.trainparam.lr was set to 0.0001. The iteration number net.trainpara. epochs was set to 1000. The RSME was selected as the performance function. The BP neural network is trained according to the above settings until it reaches the predefined target.

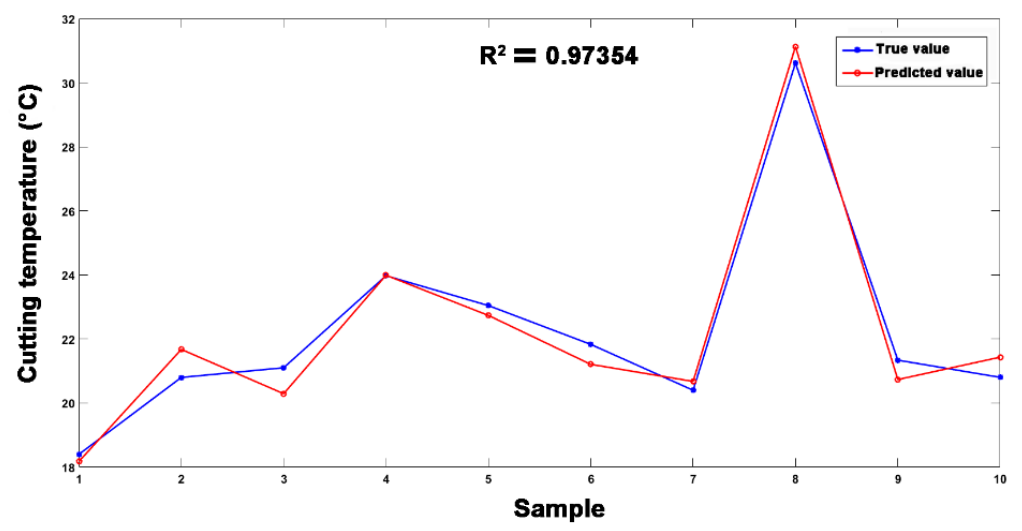

Fig. 9. Comparison of actual and predicted cutting temperatures 
Comparison of actual and predicted cutting temperatures is shown in Fig. 9, and the predicted error percentage is shown in Fig. 10. It can be learned from Fig. 9 that the predicted data was close to the test data, and the value of $\mathrm{R}^{2}$ was 0.97354 . From Fig. 10, it can be found that the maximum prediction errors were less than $4.5 \%$. According to these conclusions, the established BP neural network prediction model achieved high accuracy, therefore, it can be used to predict the cutting temperature of WPC.

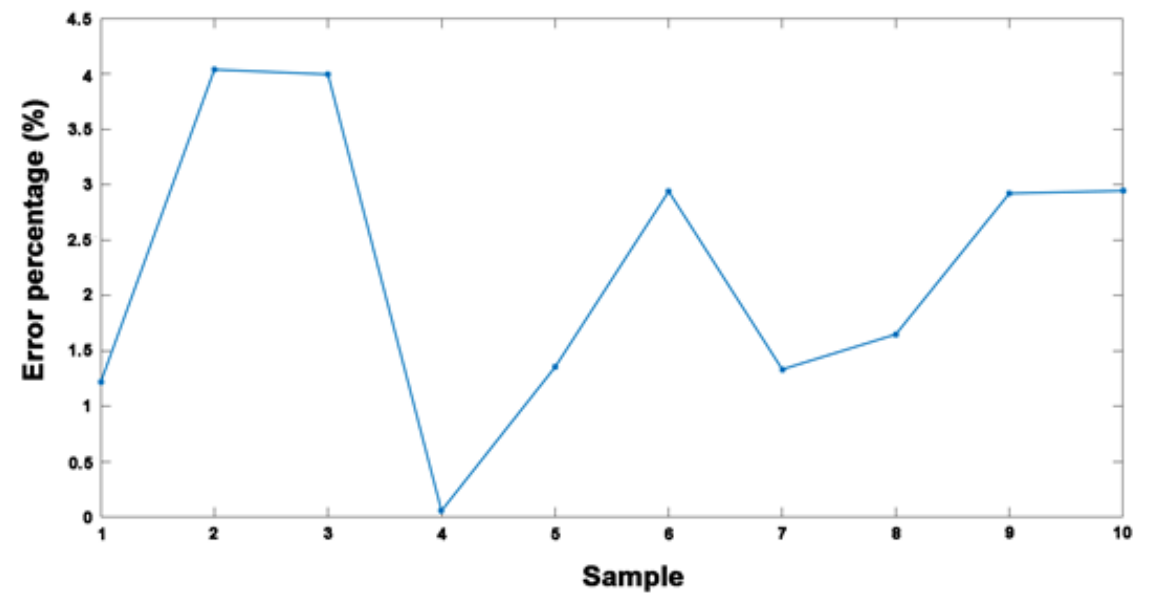

Fig. 10. Predicted error percentage

\section{CONCLUSIONS}

The experimental measurement method of cutting temperature of wood-plastic composites is described. The effect of different cutting parameters (spindle speed, feed rate, and cutting depth) on the cutting temperature of wood fiber polyethylene composite (WPC) was analyzed, and the cutting temperature prediction model using BP neural network were developed based on the experimental data. The main conclusions were the following:

1. A complete cutting cycle of WPC includes the cutting phase and non-cutting phase. In the cutting stage, cutting temperature rises rapidly at the beginning, then there are some fluctuations and the extent of fluctuation varies with different cutting parameters. In the non-cutting phase, the temperature in the cutting zone drops at a relatively slow rate; finally, it reaches a stable value.

2. The analysis of cutting temperatures revealed that the cutting temperature increases with the increase of spindle speed and cutting depth but decreases with the increase of feed rates. Therefore, in order to reduce the temperature of the cutting area in the actual cutting process, the spindle speed and cutting depth can be appropriately reduced, and the feed rate can be appropriately increased. At the same time, the cooling air flow can be adopted to reduce the cutting temperature.

3. The BP neural network was evaluated in terms of its prediction capability. The predicted values were close to the experimental data. The $\mathrm{R}^{2}$ value was calculated as 0.97354 for the testing data.

4. Within the range of cutting parameters $(6000 \leq n \leq 12000,3 \leq U \leq 6,0.4 \leq h \leq 1.6)$, the BP 
neural network prediction model achieved a high prediction accuracy. Therefore, the use of BP neural network is recommended to predict the cutting temperature of WPC without complex, expensive, and time-consuming experimental studies.

\section{Author Contribution}

All authors contributed equally to the generation and analysis of experimental data, and the development of the manuscript.

\section{ACKNOWLEDGEMENTS}

This work was supported by the National Natural Science Foundation of China [grant number 31971594].

\section{REFERENCES CITED}

Ba, D., and Boyac, S. H. (2007). "Modeling and optimization I: Usability of response surface methodology," Journal of Food Engineering 78(3), 836-845. DOI:

10.1016/j.jfoodeng.2005.11.024

Cao, D., and Guo, X. (2013). "Prediction of cutting force of austempered ductile iron based on BP neural network," in: Advanced Technologies in Manufacturing, pp. 10681074. DOI: 10.4028/www.scientific.net/AMR.774-776.1068

Chen, Z., Huang, A., and Qiang, X. (2020). "Improved neural networks based on genetic algorithm for pulse recognition," Computational Biology and Chemistry 88, article ID 107315. DOI: 10.1016/j.compbiolchem.2020.107315

Fuat, K., Aslantas, K., and Cicek, A. (2016). "Prediction of cutting temperature in orthogonal machining of AISI 316L using artificial neural network," Applied Soft Computing 38, 64-74. DOI: 10.1016/j.asoc.2015.09.034

Gardner, D. J., and Bozo, A. (2018). "Ten-year field study of wood plastic composites in Santiago, Chile: Biological, mechanical and physical property performance,"

Maderas. Ciencia y Tecnología 20(2), 257-266. DOI: 10.4067/s0718221x2018005002901

Gong, M., Sun, M., Huang, M., and Xiang, S. (2012). "Prediction of cutting consumption based on optimization-making RBF artificial neural network," in: Frontiers of Advanced Materials and Engineering Technology, pp. 659-663. DOI: 10.4028/www.scientific.net/AMR.430-432.659

Gunst, R. F. (2008). "Response surface methodology: Process and product optimization using designed experiments," Technometrics 38(3), 284-286.

Guo, X., Deng, M., Yong, H., Wang, Y., and Te, Tian (2021a). "Morphology, mechanism and kerf variation during $\mathrm{CO}_{2}$ laser cutting pine wood," Journal of Manufacturing Processes 68(Part A), 13-22. DOI: 10.1016/j.jmapro.2021.05.036

Guo, X., Wang, J., Buck, D., Zhu, Z., and Guo, Y. (2021b). "Machinability of wood fiber/polyethylene composite during orthogonal cutting," Wood Science and Technology 55, 521-534. DOI: 10.1007/s00226-020-01256-4

Guo, X., Zhang, Q., Zhu, Z., and Qing, Z. (2018). "Effect of cutting speed on cutting surface quality and tool wear during the milling of decorative particleboard using $\mathrm{CrN}$ 
coated tool," Journal of Central South University of Forestry \& Technology 38(10), 112-116. DOI: 10.14067/j.cnki.1673-923x.2018.10.017

Karaguzel, U., Bakkal, M., and Budak, E. (2016). "Modeling and measurement of cutting temperatures in milling," Procedia CIRP 46, 173-176. DOI:

10.1016/j.procir.2016.03.182

Kumar, V., Tyagi, L., and Sinha, S. (2011). "Wood flour-reinforced plastic composites: A review," Reviews in Chemical Engineering 27, 253-264. DOI:

10.1515/REVCE.2011.006

Li, Y., and Zhang, L. (2017). "A nonlinear Lagrangian method based on Log-Sigmoid function for nonconvex semidefinite programming," Journal of Industrial \& Management Optimization 5(3), 651-669. DOI: 10.3934/jimo.2009.5.651

Liukko, T., Salila, T., Platt, S., and Karki, T. (2007). "Wood plastic composites in Europe: An introduction to wood plastic composite markets and products," Baltic Forestry 13(1), 131-136.

Liyana-Pathirana, C., and Shahidi, F. (2005). "Optimization of extraction of phenolic compounds from wheat using response surface methodology," Food Chemistry 93(1), 47-56. DOI: 10.1016/j.foodchem.2004.08.050

Ma, Y., He, H., Huang, B., Jing, H., and Zhao, Z. (2020). "In situ fabrication of wood flour/nano silica hybrid and its application in polypropylene-based wood-plastic composites," Polymer Composites 41(2), 573-584. DOI: 10.1002/pc.25389

Malkapuram, R., Kumar, V., and Negi, Y. S. (2009). "Recent development in natural fiber reinforced polypropylene composites," Journal of Reinforced Plastics and Composites 28(10), 1169-1189. DOI: 10.1177/0731684407087759

Mia, M., and Dhar, N. R. (2016). "Response surface and neural network based predictive models of cutting temperature in hard turning," Journal of Advanced Research 7(6), 1035-1044. DOI: 10.1016/j.jare.2016.05.004

Nie, S. Z., and Bangyan, Y. (2009). "The application of BP neural network model of DNA-based genetic algorithm to monitor cutting tool wear," in: 2009 International Conference on Measuring Technology and Mechatronics Automation, Zhangjiajie, China, pp. 338-341. DOI: 10.1109/ICMTMA.2009.160

Patan, K. (2019). "Iterative learning control," in: Robust and Fault-Tolerant Control, Springer, Cham, Switzerland, pp. 169-201. DOI: 10.1007/978-3-030-11869-3_6

Pei, Z., Zhu, N., and Gong, Y. (2016). "A study on cutting temperature for wood-plastic composite," Journal of Thermoplastic Composite Materials 29(12), 1627-1640. DOI: $10.1177 / 0892705715570988$

Ramesh, R. S., Sadashivappa, K., and Sharanaprabhu, L. (2018). "Physical and mechanical properties: Hot pressed phenol formaldehyde based wood plastic composite, Materials Today: Proceedings 5(11, Part 3), 25331-25340. DOI: 10.1016/j.matpr.2018.10.336

Soler, D., Aristimuo, P. X., Garay, A., Arrazola, P. J., Klocke, F., Veselovac, D., and Seimann, M. (2015). "Finding correlations between tool life and fundamental dry cutting tests in finishing turning of steel," Procedia Engineering 132, 615-623. DOI: 10.1016/j.proeng.2015.12.539

Somsakova, Z., Zajac, J., Michalik, P., and Kasina, M. (2012). "Machining of wood plastic composite (pilot experiment)," Materiale Plastice 49(1), 55-57.

Sreejith, P., Krishnamurthy, R., and Malhotra, S. K. (2007). "Effect of specific cutting pressure and temperature during machining of carbon/phenolic ablative composite using PCBN tools," Journal of Materials Processing Technology 183(1), 88-95. DOI: 
10.1016/j.jmatprotec.2006.10.003

Wang, B., Wang, D., Bi, L., and Wang, H. (2020). "Surface roughness prediction based on surface temperature and tool vibration using bp neural network on turning machine," Journal of the Chinese Society of Mechanical Engineers 41(5), 615-622.

Zhu, Z., Buck, D., Guo, X., and Cao, P. (2020). "High-quality and high-efficiency machining of stone-plastic composite with diamond helical cutters," Journal of Manufacturing Processes 58, 914-922. DOI: 10.1016/j.jmapro.2020.09.004

Zhu, Z., Guo, X., Ekevad, M., Cao, P., Na, B., and Zhu, N. (2017). "The effects of cutting parameters and tool geometry on cutting forces and tool wear in milling high-density fiberboard with ceramic cutting tools," The International Journal of Advanced Manufacturing Technology 91, 4033-4041. DOI: 10.1007/s00170-017-0085-8

Article submitted: June 11, 2021; Peer review completed: August 21, 2021; Revised version received and accepted: August 30, 2021; Published: September 1, 2021. DOI: 10.15376/biores.16.4.6993-7005 